\title{
Material parameter extraction for terahertz time-domain spectroscopy using fixed-point iteration
}

\author{
W. Withayachumnankul, B. Ferguson, T. Rainsford, S. P. Mickan, and D. Abbott \\ Centre for Biomedical Engineering (CBME) and Department of Electrical \& \\ Electronic Engineering, The University of Adelaide 5005, Australia.
}

\begin{abstract}
A simple method to extract the far-infrared dielectric parameters of a homogeneous material from terahertz signals is explored in this paper. Provided with a reference, sample-probing terahertz signal and a known sample thickness, the method can determine the underlying complex refractive index of the sample within a few iterations based on the technique of fixed-point iteration. The iterative process is guaranteed to converge and gives the correct parameters when the material thickness exceeds $200 \mu \mathrm{m}$ at a frequency of $0.1 \mathrm{THz}$ or $20 \mu \mathrm{m}$ at a frequency of $1.0 \mathrm{THz}$.
\end{abstract}

Keywords: Terahertz time-domain spectroscopy, T-rays, material parameter extraction, fixed-point iteration

\section{INTRODUCTION}

Terahertz or T-ray radiation lies in the frequency range from $100 \mathrm{GHz}$ to $10 \mathrm{THz}$, and has emerged to fill the gap between the upper limit of electronics and the lower limit of the photonics. There are many promising applications including medical diagnosis, industrial quality control, airport security, and so on. ${ }^{1,2}$ One of the most widelyused applications is materials characterization using terahertz time-domain spectroscopy (THz-TDS). With the coherent and ultra-wide bandwidth nature of a single-cycle terahertz pulse, we can extract amplitude and phase information from the signal at each individual frequency. This information leads to a specific material parameter, a complex refractive index, which becomes useful in describing and distinguishing many materials. A number of solid, liquid, and gas samples have been investigated by THz-TDS, for example glass, ${ }^{3}$ crystals, ${ }^{4}$ water, ${ }^{5}$ polar gases and gas mixtures. ${ }^{6}$ Of these, semiconductors have been most extensively studied, resulting from the trend of electronics operating toward the sub-terahertz band..$^{7-9}$

Many parameter extraction methods for homogeneous materials based on a measurement of reference and probing terahertz signals have been proposed. The method introduced by Duvillaret, et al. ${ }^{3}$ models an error from the difference between estimated and measured data at each frequency. The error is then approximated by a paraboloid, and the complex refractive index at the apex of the paraboloid is found by a complicated numerical solution. Duvillaret, et al. ${ }^{10}$ and Dorney, et al. ${ }^{7}$ suggest a similar process for estimating the sample's thickness. The process simultaneously determines the set of refractive indexes at various guessed thicknesses, and uses the criterion of peak-to-peak or deepest total variation of the indexes to select the correct thickness. This is applicable when the thickness of the sample is uncertain.

The method in this paper is derived from a regular fixed-point iteration method. Provided with the reference and probing terahertz signals and the sample's thickness, the method gives a simple solution to the problem. Our solution is mathematically convergent at moderate or higher sample thicknesses. However, for a sample, which has a thickness comparable to or thinner than a wavelength of the terahertz wave, i.e. less than $300 \mu \mathrm{m}$, the sensitivity of terahertz time-domain spectroscopy is usually inadequate and it is then possible to use a more sensitive system exploiting terahertz differential time-domain spectroscopy instead. ${ }^{11,12}$

The paper is organized as follows. In Section 2 a model of the terahertz signal propagating in a planar homogeneous material and a model of material's transfer function is developed. This leads to the use of the

W. Withayachumnankul, Email: withawatw@yahoo.com; B. Ferguson, Email: bradleyferguson@yahoo.com; T. Rainsford, Email: tamath@eleceng.adelaide.edu.au; S. P. Mickan, Email: spmickan@eleceng.adelaide.edu.au; D. Abbott, Email: dabbott@eleceng.adelaide.edu.au 
fixed-point iteration method to extract parameters of a sample in Section 3. The method is carefully investigated to find a condition for convergence in Section 4. Section 5 discusses the Fabry-Pérot effect and the existing cancelation process, which could be used in conjunction with our proposed method. Two signal preprocessing techniques employed to reduce the effect of noise are explained in Section 6. The method is used to extract the parameters of a low-resistivity silicon wafer and the results are illustrated in Section 7.

\section{MODELING OF SIGNAL PROPAGATION IN HOMOGENEOUS MATERIAL}

If a plane wave, $E(\omega)$, traveling in free air, impinges on a homogeneous material at normal incidence, perpendicular to a surface of the material, the received electromagnetic wave is described by ${ }^{3}$

$$
E_{\text {sample }}(\omega)=\eta \cdot t_{\text {air,sample }} \cdot p_{\text {sample }}(\omega, L) \cdot t_{\text {sample,air }} \cdot \sum_{k=0}^{+\infty}\left\{r_{\text {sample,air }}^{2} \cdot p_{\text {sample }}^{2}(\omega, L)\right\}^{k} \cdot E(\omega),
$$

where $\eta$ is the transmission coefficient of free air, $t_{a, b}=\frac{2 \tilde{n}_{a}}{\tilde{n}_{a}+\tilde{n}_{b}}$ is the transmission coefficient from medium $a$ to medium $b, r_{a, b}=\frac{\tilde{n}_{a}-\tilde{n}_{b}}{\tilde{n}_{a}+\tilde{n}_{b}}$ is the reflection coefficient at the interface of medium $a$ and $b, p_{a}(\omega, L)=\exp \left[-i \tilde{n}_{a} \frac{\omega L}{c}\right]$ is the attenuation factor in medium $a$ over distance $L$, and $\tilde{n}_{a}=n_{a}-i \kappa_{a}$ is the frequency-dependent complex refractive index of medium $a$. The complex refractive index contains two components: $n_{a}$ is a real refractive index and $\kappa_{a}$ is an absorption index.

In the case that the plane wave travels an identical path without the presence of the sample, a received or reference wave is given by

$$
E_{\mathrm{ref}}(\omega)=\eta \cdot p_{\text {air }}(\omega, L) \cdot E(\omega) \text {. }
$$

A transfer function of the sample is obtained by deconvolving the received spectrum with respect to the reference spectrum or

$$
H(\omega)=\frac{E_{\text {sample }}(\omega)}{E_{\text {ref }}(\omega)}=\frac{4 \tilde{n}_{\text {sample }} \tilde{n}_{\text {air }}}{\left(\tilde{n}_{\text {sample }}+\tilde{n}_{\text {air }}\right)^{2}} \cdot \exp \left[-i\left(\tilde{n}_{\text {sample }}-\tilde{n}_{\text {air }}\right) \frac{\omega L}{c}\right] \cdot \operatorname{FP}(\omega) .
$$

FP $(\omega)$ represents the Fabry-Pérot effect or the interference in the received signal from reflections within the material,

$$
\operatorname{FP}(\omega)=\sum_{k=0}^{+\infty}\left\{r_{\text {sample }, \text { air }}^{2} \cdot p_{\text {sample }}^{2}(\omega, L)\right\}^{k}=\frac{1}{1-\left(\frac{\tilde{n}_{\text {sample }}-\tilde{n}_{\text {air }}}{\tilde{n}_{\text {sample }}+\tilde{n}_{\text {air }}}\right)^{2} \cdot \exp \left[-2 i \tilde{n}_{\text {sample }} \frac{\omega L}{c}\right]} .
$$

\section{PARAMETER EXTRACTION USING FIXED-POINT ITERATION}

To find the complex refractive index of the sample, the reflections or $\operatorname{FP}(\omega)$ in Equation (3) must be eliminated. Some procedures to handle this effect are given in Section 5. Assuming that preprocessing has taken place to remove the reflections from the measured signal, Equation 3 is rewritten as

$$
\begin{aligned}
H_{\text {meas }}(\omega) & =\frac{4 \tilde{n}_{\text {sample }} \tilde{n}_{\text {air }}}{\left(\tilde{n}_{\text {sample }}+\tilde{n}_{\text {air }}\right)^{2}} \cdot \exp \left[-i\left(\tilde{n}_{\text {sample }}-\tilde{n}_{\text {air }}\right) \frac{\omega L}{c}\right] \\
& =\frac{4 \tilde{n}_{\text {sample }} \tilde{n}_{\text {air }}}{\left(\tilde{n}_{\text {sample }}+\tilde{n}_{\text {air }}\right)^{2}} \cdot \exp \left[-i\left(n_{\text {sample }}-n_{\text {air }}\right) \frac{\omega L}{c}\right] \cdot \exp \left[-\kappa_{\text {sample }} \frac{\omega L}{c}\right],
\end{aligned}
$$

noting that the absorption index of air or $\kappa_{\text {air }}$ is approximately zero. From above equation, we rearrange it into the form of $n_{\text {sample }}=g_{1}\left(n_{\text {sample }}, \kappa_{\text {sample }}\right)$. Taking the argument of Equation 5 gives

$$
\arg \left[H_{\text {meas }}(\omega)\right]=\arg \left[\frac{4 \tilde{n}_{\text {sample }} \tilde{n}_{\text {air }}}{\left(\tilde{n}_{\text {sample }}+\tilde{n}_{\text {air }}\right)^{2}}\right]-\left(n_{\text {sample }}-n_{\text {air }}\right) \frac{\omega L}{c} .
$$

Hence,

$$
n_{\text {sample }}=g_{1}\left(n_{\text {sample }}, \kappa_{\text {sample }}\right)=-\frac{c}{\omega L}\left\{\arg \left[H_{\text {meas }}(\omega)\right]-\arg \left[\frac{4 \tilde{n}_{\text {sample }} \tilde{n}_{\text {air }}}{\left(\tilde{n}_{\text {sample }}+\tilde{n}_{\text {air }}\right)^{2}}\right]\right\}+n_{\text {air }} .
$$




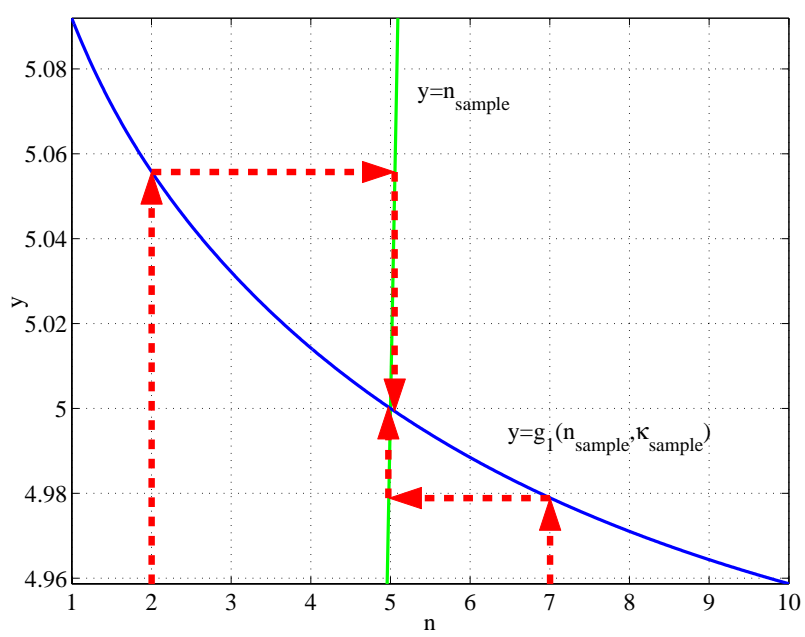

(a) Path of convergent for $g_{1}\left(n_{\text {sample }}, \kappa_{\text {sample }}\right)$ when $n_{\text {sample }}$ starts at 2 and 7 , and has a final value at 5 . Noting that $\kappa_{\text {sample }}$ is fixed at 2.5

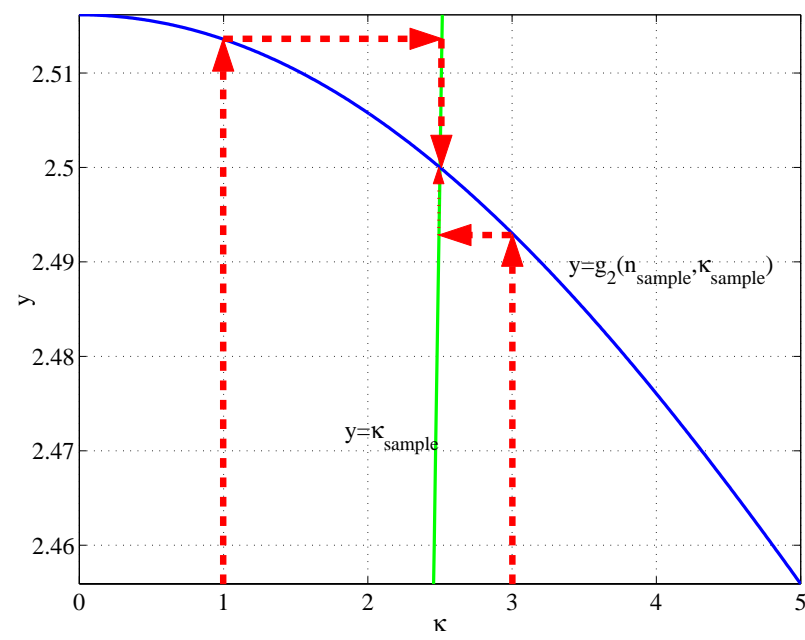

(b) Path of convergent for $g_{2}\left(n_{\text {sample }}, \kappa_{\text {sample }}\right)$ when $\kappa_{\text {sample }}$ starts at 1 and 3 , and has a final value at 2.5 . Noting that $n_{\text {sample }}$ is fixed at 5

Figure 1. Fixed-point iteration shows the convergence of functions when $\frac{\omega L}{c}=3$ and $n_{\text {sample }}-\kappa_{\text {sample }}=5-2.5 i$.

Also, we can find $\kappa_{\text {sample }}=g_{2}\left(n_{\text {sample }}, \kappa_{\text {sample }}\right)$ from Equation 5. Taking the logarithm of the absolute value of Equation 5 gives

$$
\ln \left|H_{\text {meas }}(\omega)\right|=\ln \left|\frac{4 \tilde{n}_{\text {sample }} \tilde{n}_{\text {air }}}{\left(\tilde{n}_{\text {sample }}+\tilde{n}_{\text {air }}\right)^{2}}\right|-\kappa_{\text {sample }} \frac{\omega L}{c} .
$$

Hence,

$$
\kappa_{\text {sample }}=g_{2}\left(n_{\text {sample }}, \kappa_{\text {sample }}\right)=-\frac{c}{\omega L}\left\{\ln \left|H_{\text {meas }}(\omega)\right|-\ln \left|\frac{4 \tilde{n}_{\text {sample }} \tilde{n}_{\text {air }}}{\left(\tilde{n}_{\text {sample }}+\tilde{n}_{\text {air }}\right)^{2}}\right|\right\} .
$$

Equations 7 and 9 are in the form of a fixed-point iteration or $x=g(x)$ method in two dimensions. ${ }^{13}$ Provided the initial value of $n_{\text {sample }}$ and $\kappa_{\text {sample }}$ for the complex refractive index, the function $g_{1}\left(n_{\text {sample }}, \kappa_{\text {sample }}\right)$ and $g_{2}\left(n_{\text {sample }}, \kappa_{\text {sample }}\right)$ maps those points to new points. Repeatedly substituting the new values of $n_{\text {sample }}$ and $\kappa_{\text {sample }}$ into both equations gives updated values. If the functions appear convergent, when there is small change of $n_{\text {sample }}$ and $\kappa_{\text {sample }}$ over the succeeding iterations, $n_{\text {sample }}$ and $\kappa_{\text {sample }}$ are then called fixed points of the functions.

If initial values of $n_{\text {sample }}$ and $\kappa_{\text {sample }}$ or $n_{0}$ and $\kappa_{0}$ are selected to be close to the final values, the iteration times can be reduced to some extent. Therefore, from Equation 7 and 9 when $\frac{4 \tilde{n}_{\text {sample }} \tilde{n}_{\text {air }}}{\left(\tilde{n}_{\text {sample }}+\tilde{n}_{\text {air }}\right)^{2}}=1, n_{0}$ and $\kappa_{0}$ are given by

$$
\begin{aligned}
n_{0} & =-\frac{c}{\omega L} \arg \left[H_{\text {meas }}(\omega)\right]+n_{\text {air }}, \\
\kappa_{0} & =-\frac{c}{\omega L} \ln \left|H_{\text {meas }}(\omega)\right|
\end{aligned}
$$

\section{CONVERGENCE OF THE METHOD}

To illustrate how the function $n_{\text {sample }}=g_{1}$ converges, two subfunctions, $y=n_{\text {sample }}$ and $y=g_{1}$, are plotted on the same axis. The final solution lies where these two functions cross each other. Figure 1a shows the plot of subfunctions $y=n_{\text {sample }}$ and $y=g_{1}$ when $\kappa_{\text {sample }}$ is fixed to 2.5. For example, starting at initial value $n_{\text {sample }}=7$, the function $y=g_{1}$ iterates it to the new value at 4.98. This new value is iterated back by $y=n_{\text {sample }}$ to $y=g_{1}$, providing the value 4.999 , which is closer to the final solution at $n_{\text {sample }}=5$. The same 


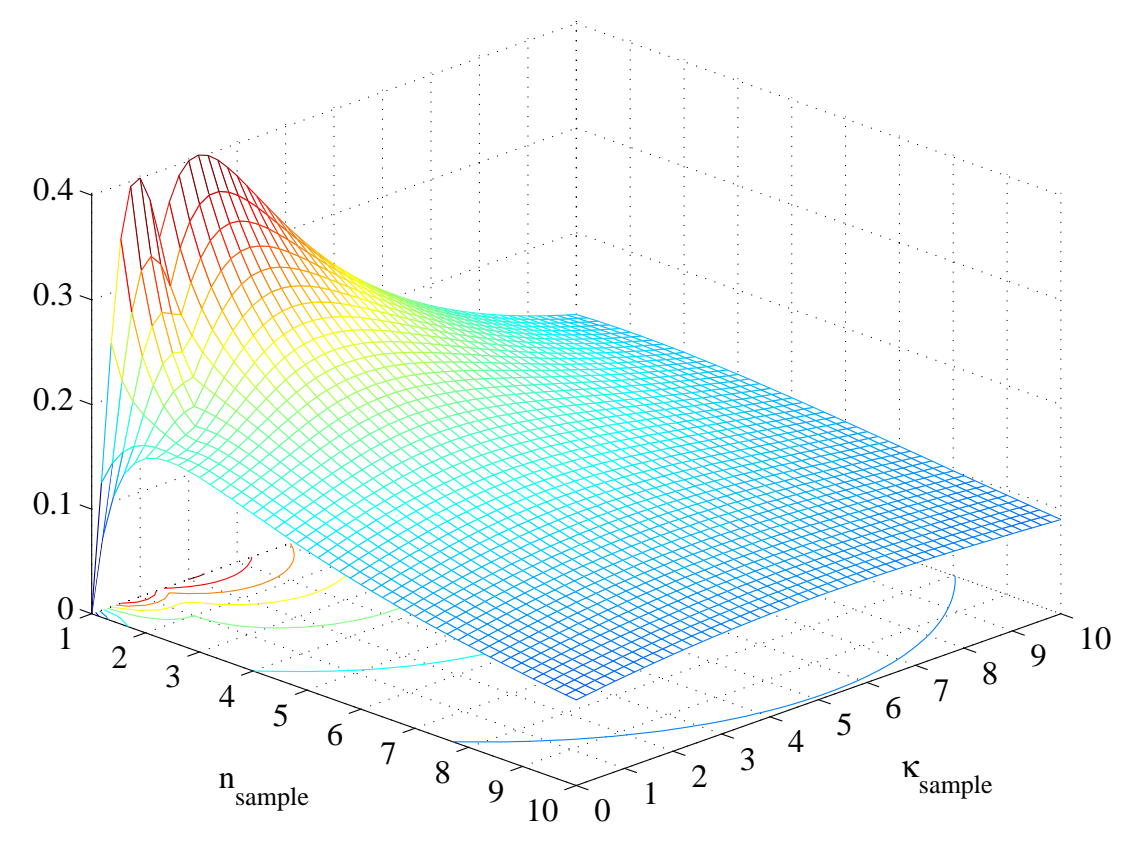

Figure 2. Plotting of $\left|\frac{\partial g_{1}}{\partial n_{\text {sample }}}\right|+\left|\frac{\partial g_{1}}{\mathrm{~d} \kappa_{\text {sample }}}\right|$ when $\frac{\omega L}{c}=1$ over the region of interest.

mechanism also appears in the subfunctions $y=\kappa_{\text {sample }}$ and $y=g_{2}$ in Figure $1 \mathrm{~b}$. Observe that the functions converge to correct solutions in a few iterations.

Given that both fixed-point functions, $n_{\text {sample }}=g_{1}\left(n_{\text {sample }}, \kappa_{\text {sample }}\right)$ and $\kappa_{\text {sample }}=g_{2}\left(n_{\text {sample }}, \kappa_{\text {sample }}\right)$, are first-order differentiable, two sufficient conditions to guarantee the convergence of the functions are

$$
\left|\frac{\partial g_{1}}{\partial n_{\text {sample }}}\right|+\left|\frac{\partial g_{1}}{\mathrm{~d} \kappa_{\text {sample }}}\right|<1
$$

and

$$
\left|\frac{\partial g_{2}}{\partial n_{\text {sample }}}\right|+\left|\frac{\partial g_{2}}{\partial \kappa_{\text {sample }}}\right|<1
$$

along the search path i.e. from the initial value to the fixed point. When the summation of the absolute value is close to zero, the function converges rapidly.

A partial derivative of $g_{1}$ with respect to $n_{\text {sample }}$ is given by

$$
\frac{\partial g_{1}}{\partial n_{\text {sample }}}=\frac{c}{\omega L} \cdot\left\{\frac{\kappa_{\text {sample }}}{n_{\text {sample }}^{2}+\kappa_{\text {sample }}^{2}}-\frac{2 \kappa_{\text {sample }}}{\left(n_{\text {sample }}+n_{\text {air }}\right)^{2}+\kappa_{\text {sample }}^{2}}\right\},
$$

whereas a partial derivative of $g_{1}$ with respect to $\kappa_{\text {sample }}$ is given by

$$
\frac{\partial g_{1}}{\partial \kappa_{\text {sample }}}=\frac{c}{\omega L} \cdot\left\{-\frac{n_{\text {sample }}}{n_{\text {sample }}^{2}+\kappa_{\text {sample }}^{2}}+\frac{2\left(n_{\text {sample }}+n_{\text {air }}\right)}{\left(n_{\text {sample }}+n_{\text {air }}\right)^{2}+\kappa_{\text {sample }}^{2}}\right\} .
$$

Substituting these two derivatives into Equation 11 gives

$$
\left|\frac{\kappa_{\text {sample }}}{n_{\text {sample }}^{2}+\kappa_{\text {sample }}^{2}}-\frac{2 \kappa_{\text {sample }}}{\left(n_{\text {sample }}+n_{\text {air }}\right)^{2}+\kappa_{\text {sample }}^{2}}\right|+\left|\frac{n_{\text {sample }}}{n_{\text {sample }}^{2}+\kappa_{\text {sample }}^{2}}-\frac{2\left(n_{\text {sample }}+n_{\text {air }}\right)}{\left(n_{\text {sample }}+n_{\text {air }}\right)^{2}+\kappa_{\text {sample }}^{2}}\right|<\frac{\omega L}{c} .
$$


To validate the condition, all possible values of $n_{\text {sample }}$ and $\kappa_{\text {sample }}$ are taken into account. Normally, a practical

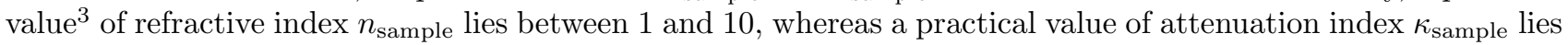
between 0 and 10. The maximum value for the LHS of Equation 15 occurs at $n_{\text {sample }}=1$ and $\kappa_{\text {sample }}=0.9234$ (see Figure 2 and Appendix A). By putting this critical point into Equation 15, the condition of convergence is

$$
0.4024<\frac{\omega L}{c} .
$$

This is not a decisive condition since the function $g_{2}$ has not yet been considered. Hence, a partial derivative of $g_{2}$ with respect to $n_{\text {sample }}$ is given by

$$
\frac{\partial g_{2}}{\partial n_{\text {sample }}}=\frac{c}{\omega L} \cdot\left\{\frac{n_{\text {sample }}}{n_{\text {sample }}^{2}+\kappa_{\text {sample }}^{2}}-\frac{2\left(n_{\text {sample }}+n_{\text {air }}\right)}{\left(n_{\text {sample }}+n_{\text {air }}\right)^{2}+\kappa_{\text {sample }}^{2}}\right\},
$$

whereas a partial derivative of $g_{2}$ with respect to $\kappa_{\text {sample }}$ is given by

$$
\frac{\partial g_{2}}{\partial \kappa_{\text {sample }}}=\frac{c}{\omega L} \cdot\left\{\frac{\kappa_{\text {sample }}}{n_{\text {sample }}^{2}+\kappa_{\text {sample }}^{2}}-\frac{2 \kappa_{\text {sample }}}{\left(n_{\text {sample }}+n_{\text {air }}\right)^{2}+\kappa_{\text {sample }}^{2}}\right\} \text {. }
$$

Substituting these two derivatives into Equation 12 gives

$\left|\frac{\kappa_{\text {sample }}}{n_{\text {sample }}^{2}+\kappa_{\text {sample }}^{2}}-\frac{2 \kappa_{\text {sample }}}{\left(n_{\text {sample }}+n_{\text {air }}\right)^{2}+\kappa_{\text {sample }}^{2}}\right|+\left|\frac{n_{\text {sample }}}{n_{\text {sample }}^{2}+\kappa_{\text {sample }}^{2}}-\frac{2\left(n_{\text {sample }}+n_{\text {air }}\right)}{\left(n_{\text {sample }}+n_{\text {air }}\right)^{2}+\kappa_{\text {sample }}^{2}}\right|<\frac{\omega L}{c}$.

It appears that the second condition in Equation 19 is identical to the first condition in Equation 15. As a result, the condition which makes the functions converge is given by Equation 16 and is valid for all practical cases.

The estimated condition is confirmed by the simulation as shown in Figure 3. Given an equation to find an error of refractive indexes,

$$
\delta_{i}=\left|n_{\mathrm{fp}}-n_{i}\right|+\left|\kappa_{\mathrm{fp}}-\kappa_{i}\right|,
$$

where $\left(n_{\mathrm{fp}}, \kappa_{\mathrm{fp}}\right)$ is a fixed point, and $\left(n_{\mathrm{i}}, \kappa_{\mathrm{i}}\right)$ is an estimated point at $i^{\text {th }}$ iteration, we can observe the characteristic of the error in the simulation. When $\frac{\omega L}{c}$ is below 0.4 , disregarding the value of complex refractive index, the

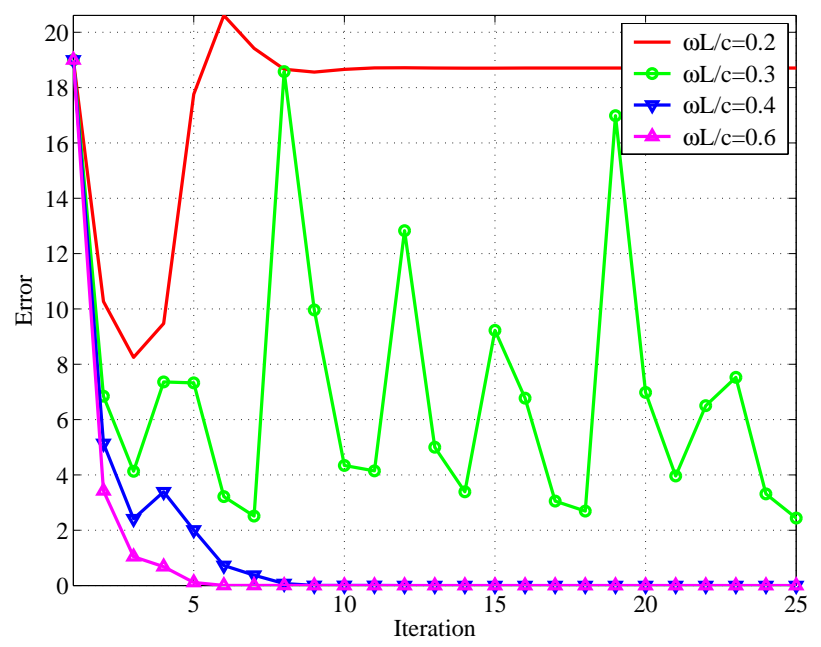

Figure 3. Change of the error over a number of iterations. The starting point and the fixed point are given by $n_{\text {sample }}-$ $\kappa_{\text {sample }}=10-10 i$ and $n_{\text {sample }}-\kappa_{\text {sample }}=1-0 i$, respectively. These two points are selected to force the search path to cross over the entire search space. 
functions diverge or oscillate. On the other hand, when $\frac{\omega L}{c}$ is set equal to or higher than the threshold, according to Equation 16, the functions become convergent. At the ninth iteration, the error when $\frac{\omega L}{c}=0.4$ is $2.3 \times 10^{-3}$, and reduces to $3.7 \times 10^{-16}$ when $\frac{\omega L}{c}=0.6$. Obviously, the simulation is in accordance with the prediction.

The condition implies that, at $f=0.1 \mathrm{THz}$, the minimum applicable thickness $L$ is $200 \mu \mathrm{m}$ and at $f=1.0 \mathrm{THz}$ the minimum applicable thickness is $20 \mu \mathrm{m}$. Figure 4 shows the plotting of the border line of convergence.

\section{FABRY-PÉROT EFFECT CANCELATION}

When the Fabry-Pérot effect occurs in the signal and is not excluded prior to parameter extraction, the method gives an incorrect result, as shown in Figure 5 . The values of $n_{\text {sample }}$ and $\kappa_{\text {sample }}$ severely oscillate at low frequencies. An extra procedure is required to reduce this problem.

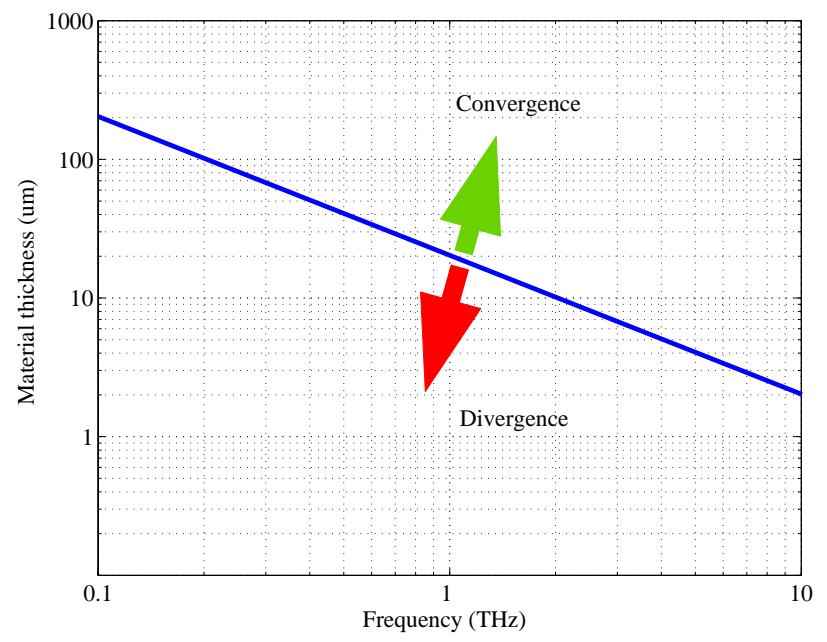

Figure 4. The borderline of convergence illustrates an appropiate thickness of material at signal frequencies from $100 \mathrm{GHz}$ to $10 \mathrm{THz}$.

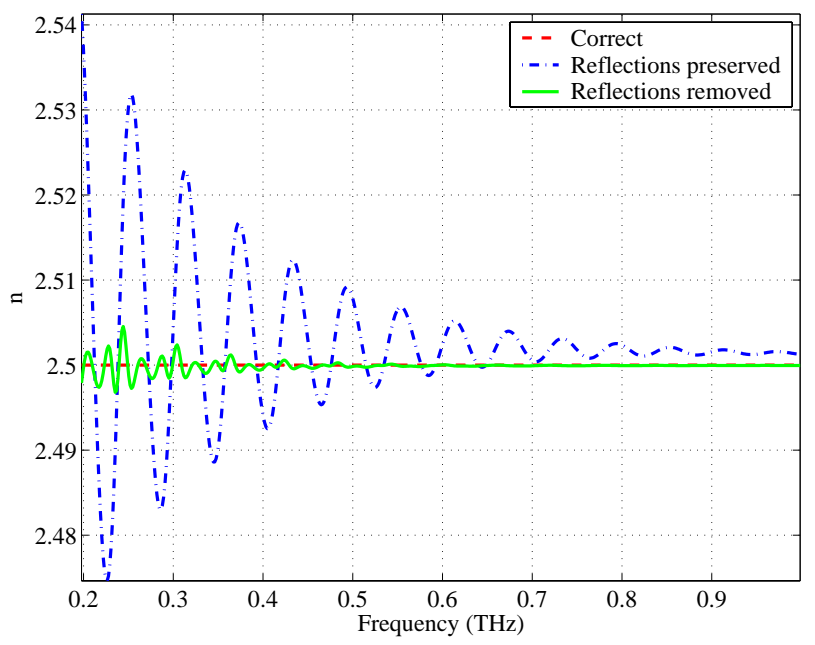

(a) Refractive index, $n$.

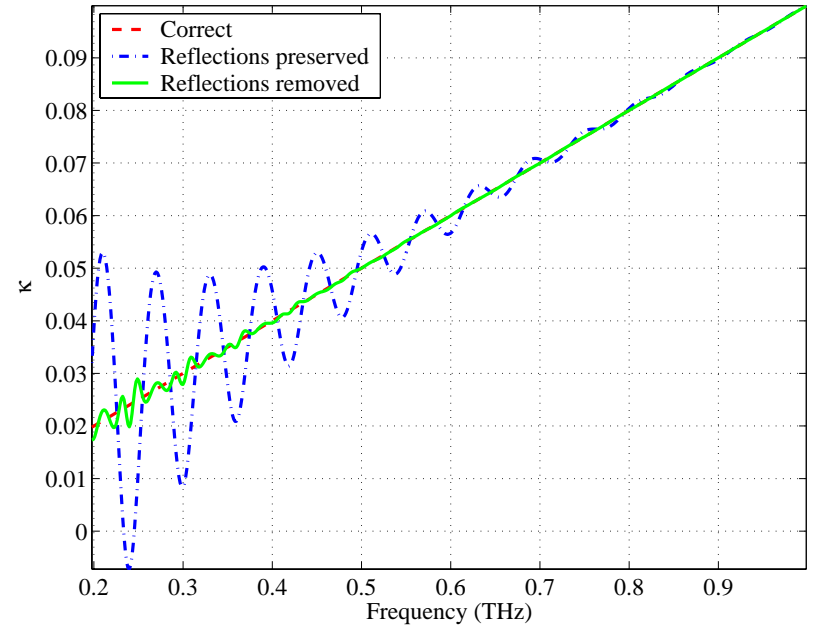

(b) Attenuation index, $\kappa$.

Figure 5. The refractive indices extracted from the signal in which the reflections are preserved and from the signal receiving the Fabry-Pérot cancelation process three times. The parameters used in this simulation are $L=1 \mathrm{~mm}$ and $n_{\text {sample }}-\kappa_{\text {sample }}=2.5-0.1 \frac{\omega}{2 \pi} i$. 


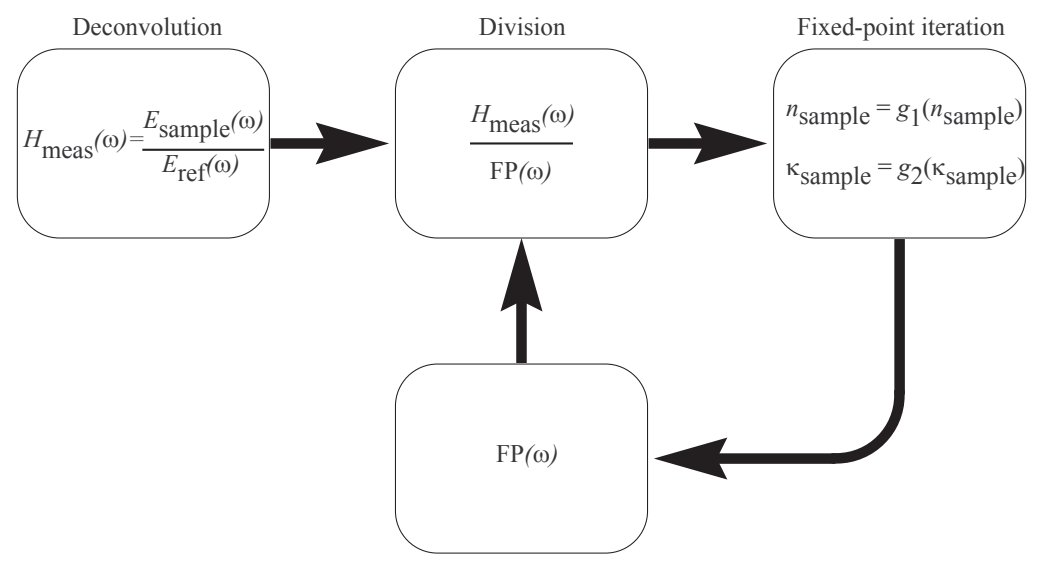

Figure 6. The Fabry-Pérot cancelation process.

A solution to this problem depends on the thickness of the sample. For an optically thick sample the reflections are easily separable from the primary received signal, in a timescale sufficient to allow a windowing technique to be implemented to pick up only the main signal. This is not the case for an optically thin sample where the reflections interfere with the primary signal. A more sophisticated procedure is required to subtract them out.

A Fabry-Pérot cancelation process was proposed by Duvillaret, et al. ${ }^{3}$ In that article it was implemented in conjunction with material parameter extraction by minimization of the error. Given the primary terahertz pulse and all of its reflections, the process can cancel out most of the effect regardless of the thickness of the sample. If the signal reflections are rejected, prior to the process, the result becomes unpredictable.

A diagram of the process is shown in Figure 6. By setting the $\operatorname{FP}(\omega)$ to 1 for the first iteration, the measured transfer function of the sample $H_{\text {meas }}(\omega)$ is put into the fixed-point iteration directly. Intermediate extracted parameters $n_{\text {sample }}$ and $\kappa_{\text {sample }}$ from the fixed-point iteration are later used to determine the $\operatorname{FP}(\omega)$ term as described by Equation 4. According to Equation 3 the Fabry-Pérot term can be canceled by dividing $H_{\text {meas }}(\omega)$ with the estimated $\operatorname{FP}(\omega)$. The updated transfer function is put into the fixed-point iteration again. Running the process repeatedly, we can obtain improved parameters $n_{\text {sample }}$ and $\kappa_{\text {sample }}$.

We set up a simulation to validate that the cancelation process can be used with our proposed parameter extraction method correctly. The material parameters are set to $L=1 \mathrm{~mm}$ and $n_{\text {sample }}-\kappa_{\text {sample }}=2.5-0.1 \frac{\omega}{2 \pi} i$. After the process runs for three iterations, the result is available as shown in Figure 5. Obviously, the oscillation previously dominating the result decreases by a large amount. Hence, the process can be successfully used in conjunction with our method to cancel out the Fabry-Pérot reflections.

\section{SIGNAL PREPROCESSING}

Terahertz signals always encounter various sources of noise, which leads to uncertainties in the extracted parameters. ${ }^{14}$ Hence, we attempt to reduce the effect of the noise prior to the parameter extraction process, using two techniques: (i) spatial windowing and (ii) extrapolated phase unwrapping.

\subsection{Spatial Windowing}

A single-cycle terahertz pulse has a high amplitude only in a very short duration compared to the very long recording period. White Gaussian noise covering the long low-amplitude portion of the signal contributes significantly to the total noise power. Therefore, the total noise power is readily decreased by applying the window to the peak period of the signal and windowing out the portion of signal elsewhere.

However, one important thing must be considered while we choose the size and position of the window. Since the Fabry-Pérot cancelation processes in Section 5 requires the reflections being intact to work properly, if this 
process is used, we must ensure that the window does remove any significant part of the reflections - only the farther reflections, having an amplitude below the noise level are excluded.

Although the simple yet effective way to lessen the effect of noise is windowing, more robust denoising techniques for terahertz signals such as wavelet thresholding ${ }^{15}$ exist but are beyond the scope of this present work.

\subsection{Phase unwrapping}

The argument of the transfer function used in Equation $7, \arg \left[H_{\text {meas }}(\omega)\right]$, is obtained from the phase unwrapping technique. The standard phase unwrapping starts from zero radian and progresses towards higher frequencies. However, an error in lower frequency phase could propagate through all remaining frequencies. And since the noise severely perturbs the signal at low frequencies, an extra step is necessary to avoid false unwrapped phase.

The adapted phase unwrapping procedure starts from the frequency at which the spectrum has the largest SNR upward to the end and downward to lower cutoff frequency. Then the phase from this point to zero radian is extrapolated from the unwrapped phase at higher frequencies. In most cases the assumption of linear phase relation is sufficient..$^{3,16}$

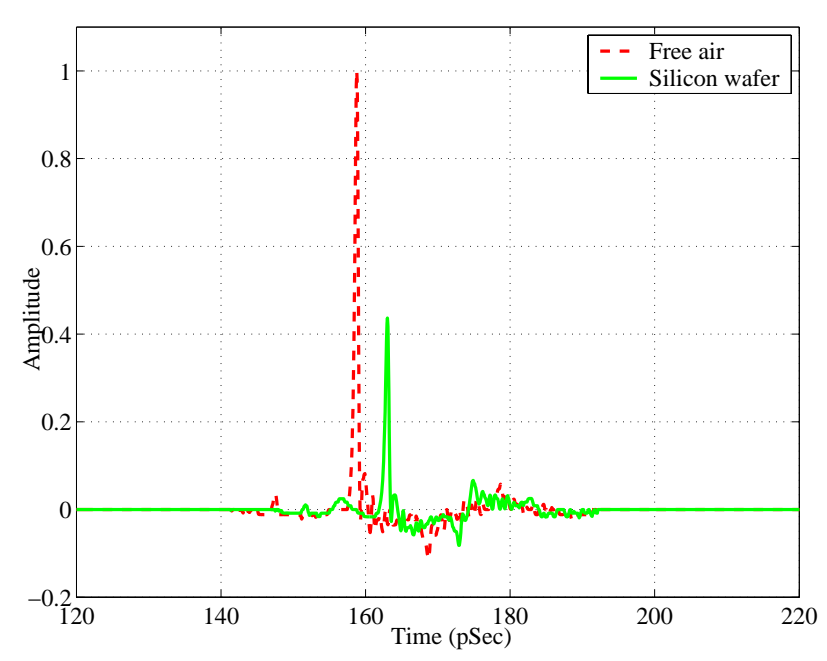

(a) Terahertz signals probing free air and silicon wafer. The amplitude of the signals are preserved in the range between 140 and $190 \mathrm{pSec}$ and nullified elsewhere.

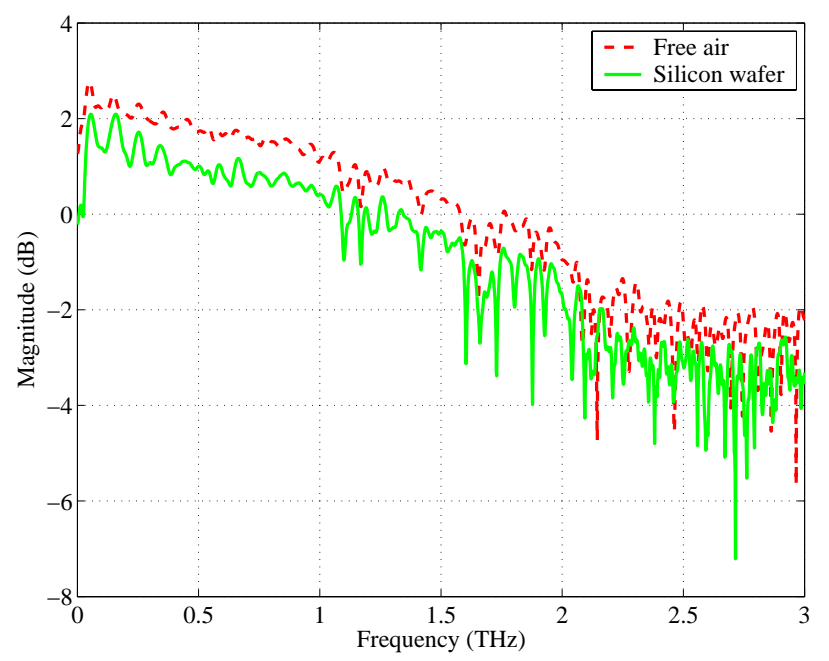

(b) Spectra of terahertz signals for free air and the silicon wafer.

Figure 7. Terahertz signals and their corresponding spectra.

\section{RESULTS}

We extract the underlying parameters of a sample from the terahertz signals using the proposed fixed-point iteration. The sample under test is a $500 \mu \mathrm{m}$-thick low resistivity silicon wafer having the characteristics as follows: (i) polished on both sides, (ii) undoped, (iii) unbiassed, (iv) $<100>$ crystal orientation, and (v) bulk silicon. Figure 7a shows the terahertz signals for free air and a silicon wafer after the $50 \mathrm{pSec}$ window is applied. The signal for the silicon wafer still contains the primary pulse and the first three reflections in order to allow the Fabry-Pérot effect cancelation process in Section 5 to work properly.

A discrete Fourier transform carried out on both the sample and reference (air) terahertz signals yields the spectra as shown in Figure 7b. Since the length of our window is $50 \mathrm{pSec}$, the frequency resolution of the spectra is approximately $20 \mathrm{GHz}$. The effective bandwidth of the transfer function, which provides reliable information, lies from 0.2 to $1.0 \mathrm{THz}$. A transfer function of the sample, $H(\omega)$, determined from the spectra, is shown in Figure 8 in the form of the logarithm of the absolute value and the unwrapped phase. 


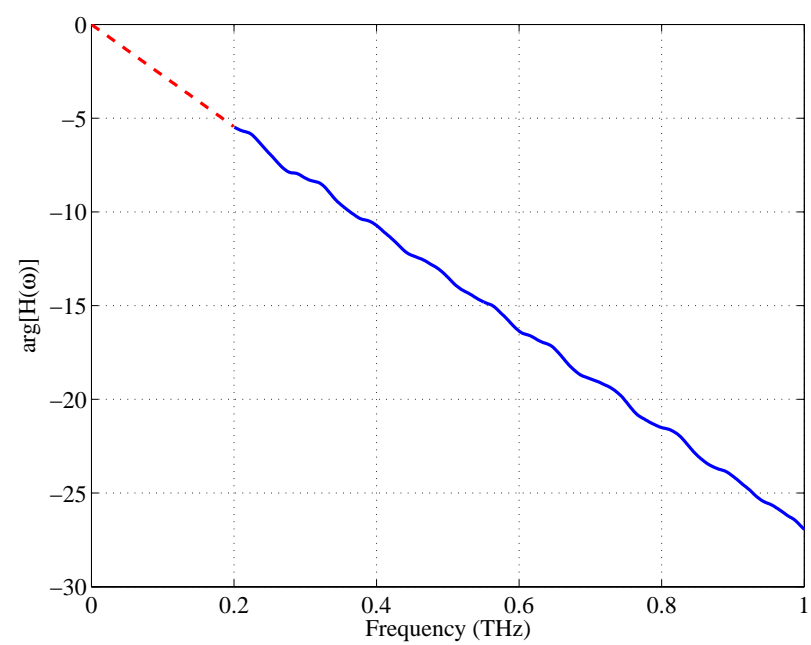

(a) Unwrapped phase or argument of the transfer function. Dotted line shows the extrapolated region of the phase.

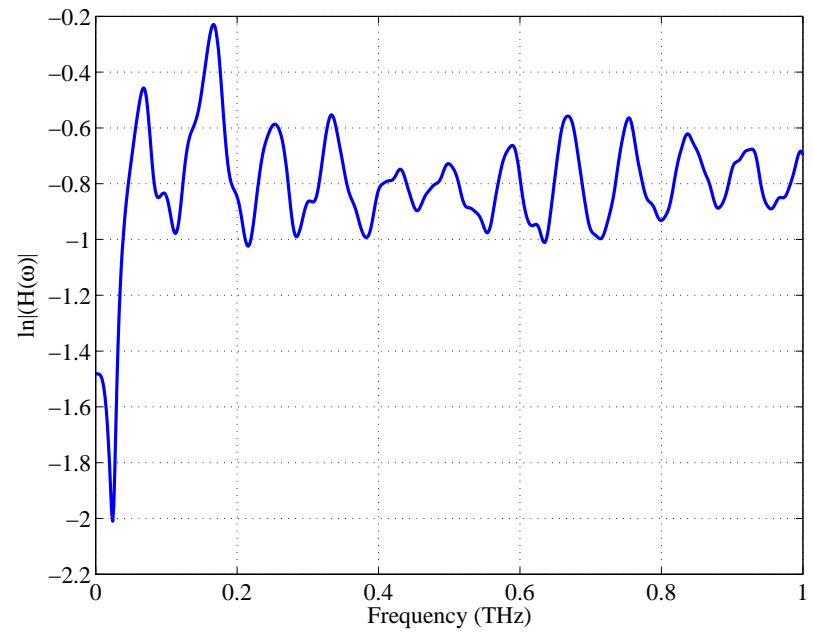

(b) Logarithm of the absolute of the transfer function.

Figure 8. Transfer function of the silicon wafer.

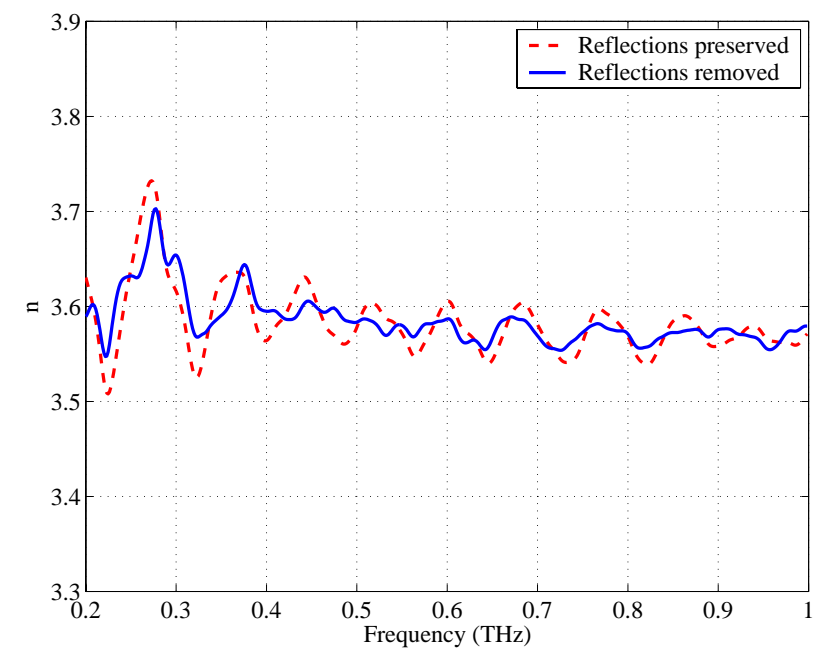

(a) Refractive index, $n$.

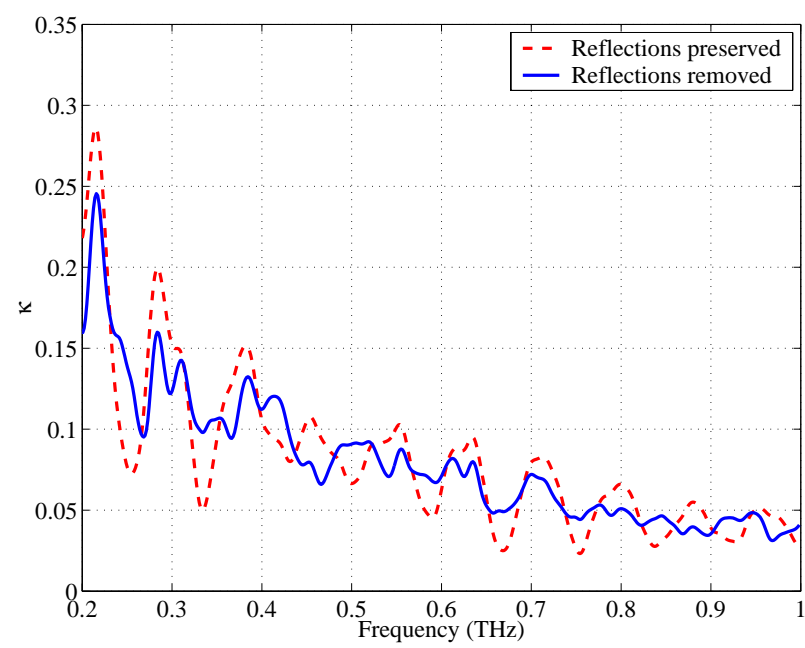

(b) Absorption index, $\kappa$.

Figure 9. The refractive index and absorption index of the low-resistivity silicon wafer before and after applying the Fabry-Pérot cancelation process.

The fixed point iteration method is applied to the measured data over the effective bandwidth with the number of iterations set to 5 at each frequency point. Figure 9 shows the extracted refractive index and absorption index before and after the Fabry-Pérot cancelation process is repeated 5 times. The results after the reflections being removed represent lower oscillation of the parameters over the frequency range. The values of the indexes coincide with those obtained from the parabolic-fit method. ${ }^{3}$

\section{CONCLUSIONS}

The parameter extraction method derived from the regular fixed-point iteration is presented. It requires two terahertz signals, a sample signal and a reference signal, and the thickness of the sample to determine the complex 
refractive index. From prediction and simulation, the method always produces a convergent solution when the thickness of sample is higher than $200 \mu \mathrm{m}$ at $0.1 \mathrm{THz}$ or $20 \mu \mathrm{m}$ at $1.0 \mathrm{THz}$, regardless of the refractive index value. This condition is applicable in most cases common to THz-TDS. In addition, the method can work in complement with the Fabry-Pérot cancelation process, which is used to remove the oscillations from extracted parameters.

Our method is validated by being tested with a real terahertz signal passing through a silicon wafer. The results concur with those obtained using a standard parameter extraction method.

Despite the limitation of the sample's thickness the method gives a simple but effective way to determine the parameters of a sample from the terahertz signal.

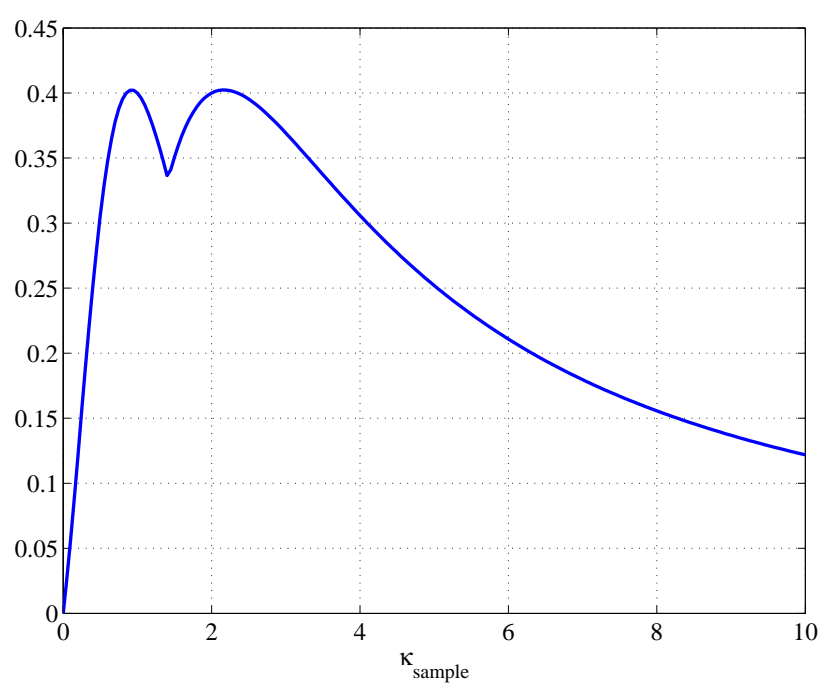

Figure 10. Plotting of $\left|\frac{\partial g_{1}}{\partial n_{\text {sample }}}\right|+\left|\frac{\partial g_{1}}{\mathrm{~d} \kappa_{\text {sample }}}\right|$ when $\frac{\omega L}{c}=1$ and $n_{\text {sample }}=1$.

\section{APPENDIX A. MAXIMUM VALUE OF THE CONDITION}

The pair of $n_{\text {sample }}$ and $\kappa_{\text {sample }}$ which gives the maximum value for the LHS of Equation 15, or

$$
\left|\frac{\kappa_{\text {sample }}}{n_{\text {sample }}^{2}+\kappa_{\text {sample }}^{2}}-\frac{2 \kappa_{\text {sample }}}{\left(n_{\text {sample }}+n_{\text {air }}\right)^{2}+\kappa_{\text {sample }}^{2}}\right|+\left|\frac{n_{\text {sample }}}{n_{\text {sample }}^{2}+\kappa_{\text {sample }}^{2}}-\frac{2\left(n_{\text {sample }}+n_{\text {air }}\right)}{\left(n_{\text {sample }}+n_{\text {air }}\right)^{2}+\kappa_{\text {sample }}^{2}}\right|,
$$

is determined by a normal procedure. Initially, the boundary or the possible range of $n_{\text {sample }}$ and $\kappa_{\text {sample }}$ is set up to limit the search space. It is given by

$$
\begin{aligned}
& 1 \leq n_{\text {sample }} \leq 10 \\
& 0 \leq \kappa_{\text {sample }} \leq 10
\end{aligned}
$$

Since the function is defined on a closed bounded interval, it definitely has a global maximum. This global maximum could be a local maximum or an endpoint maximum. However, from Figure 2 the function has no local maximum in the region of interest. We turn to an endpoint maximum at which $n_{\text {sample }}=1$. Substituting $n_{\text {sample }}=1$ to Equation 21 gives

$$
f\left(\kappa_{\text {sample }}\right)=\left|\frac{\kappa_{\text {sample }}}{1+\kappa_{\text {sample }}^{2}}-\frac{2 \kappa_{\text {sample }}}{4+\kappa_{\text {sample }}^{2}}\right|+\left|\frac{1}{1+\kappa_{\text {sample }}^{2}}-\frac{4}{4+\kappa_{\text {sample }}^{2}}\right|,
$$


or

$$
f\left(\kappa_{\text {sample }}\right)= \begin{cases}\frac{\kappa_{\text {sample }}-1}{1+\kappa_{\text {sample }}^{2}}-\frac{2 \kappa_{\text {sample }}-4}{4+\kappa_{\text {sample }}^{2}} & \text { if } 0<\kappa_{\text {sample }}<\sqrt{2} \\ -\frac{\kappa_{\text {sample }}+1}{1+\kappa_{\text {sample }}^{2}}+\frac{2 \kappa_{\text {sample }}+4}{4+\kappa_{\text {sample }}^{2}} & \text { if } \sqrt{2}<\kappa_{\text {sample }}<10\end{cases}
$$

The function in Equation 23 is plotted against $\kappa_{\text {sample }}$ as shown in Figure 10. Both sections of the function in Equation 24 are differentiated to find a critical point which leads to the maximum. It appears that the critical points locate at $\kappa_{\text {sample }}=0.9234$ and $\kappa_{\text {sample }}=2.1660$, giving the identical maximum value of $f\left(\kappa_{\text {sample }}\right)=$ 0.4024 .

\section{REFERENCES}

1. D. M. Mittleman, R. H. Jacobsen, and M. C. Nuss, "T-ray imaging," IEEE Journal of Selected Topics in Quantum Electronics 2, pp. 679-692, September 1996.

2. D. M. Mittleman, M. Gupta, R. Neelamani, R. Baraniuk, J. Rudd, and M.Koch, "Recent advances in terahertz imaging," Applied Physics B: Lasers and Optics 68, pp. 1085-1094, June 1999.

3. L. Duvillaret, F. Garet, and J. L. Coutaz, "A reliable method for extraction of material parameters in terahertz time-domain spectroscopy," IEEE Journal of Selected Topics in Quantum Electronics 2, pp. 739746, September 1996.

4. E. V. Loewenstein, D. R. Smith, and R. L. Morgan, "Optical constants of far infrared materials. 2: Crystalline solids," Applied Optics 12, pp. 398-406, February 1973.

5. C. Rønne, P.-O. Åstrand, and S. R. Keiding, "THz spectroscopy of liquid $\mathrm{H}_{2} \mathrm{O}$ and $\mathrm{D}_{2} \mathrm{O}$," Physical Review Letters 82, pp. 2888-2891, April 1999.

6. D. M. Mittleman, R. H. Jacobsen, R. Neelamani, R. G. Baraniuk, and M. C. Nuss, "Gas sensing using terahertz time-domain spectroscopy," Applied Physics B: Lasers and Optics 67, pp. 379-390, September 1998.

7. T. D. Dorney, R. G. Baraniuk, and D. M. Mittleman, "Material parameter estimation with terahertz timedomain spectroscopy," Journal of the Optical Society of America A 18, pp. 1562-1571, July 2001.

8. M. van Exter and D. Grischkowsky, "Optical and electronic properties of doped silicon from 0.1 to 2 THz," Applied Physics Letters 56, pp. 1694-1696, April 1990.

9. N. Katzenellenbogen and D. Grischkowsky, "Electrical characterization to $4 \mathrm{THz}$ of N- and P-type GaAs using thz time-domain spectroscopy," Applied Physics Letters 61, pp. 840-842, August 1992.

10. L. Duvillaret, F. Garet, and J.-L. Coutaz, "Highly precise determination of optical constants and sample thickness in terahertz time-domain spectroscopy," Applied Optics 38, pp. 409-415, January 1999.

11. Z. Jiang, M. Li, and X.-C. Zhang, "Dielectric constant measurement of thin films by differential time-domain spectroscopy," Applied Physics Letters 76, pp. 3221-3223, May 2000.

12. K.-S. Lee, T.-M. Lu, and X.-C. Zhang, "Tera tool," IEEE Circuits \& Devices Magazine 18, pp. 23-28, November 2002.

13. C. F. Gerald and P. O. Wheatley, Applied Numerical Analysis, Addison-Wesley, Massachusetts, USA, 6th ed., 1999.

14. L. Duvillaret, F. Garet, and J.-L. Coutaz, "Influence of noise on the characterization of materials by terahertz time-domain spectroscopy," Journal of the Optical Society of America B 17, pp. 452-460, March 2000.

15. B. Ferguson and D. Abbott, "Wavelet de-noising of optical terahertz pulse imaging data," Journal of Fluctuation and Noise Letters 1(2), pp. L65-L69, 2001.

16. B. Ferguson, Three Dimensional T-ray Inspection Systems. PhD thesis, School of Electrical and Electronic Engineering, Faculty of Engineering, Computer and Mathematical Sciences, The University of Adelaide, Australia, 2004. 